Approved for public release; distribution is unlimited.
Title:

USE OF RESPONSE SURFACE METAMODELS FOR DAMAGE IDENTIFICATION OF A SIMPLE NONLINEAR SYSTEM

Author(s):

Amanda L. Cundy

Francois M. Hemez

Daniel J. Inman

Gyuhae Park
DAMAS 2003, International Conference on Damage Assessment of Structures, July 1-3, 2003, University of Southampton, UK

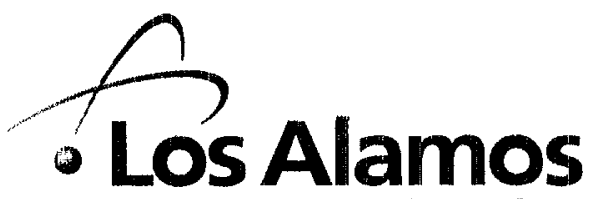

NATIONAL I.ABORATORY

Los Alamos National Laboratory, an affirmative action/equal opportunity employer, is operated D.e University of California for the U.S. Department of Energy under contract W-7405-ENG-36. By acceptance of this article, the publisher recognizes that the U.S. Government retains a nonexclusive, royalty-free license to publish or reproduce the published form of this contribution, or to allow others to do so, for U.S. Government purposes. Los Alamos National Laboratory requests that the publisher identify this article as work performed under the auspices of the U.S. Department of Energy. Los Alamos National Laboratory strongly supports academic freedom and a researcher's right to publish; as an institution, however, the Laboratory does not endorse the viewpoint of a publication or guarantee its technical correctness. 


\title{
Use of Response Surface Metamodels for Damage Identification of a Simple Non-Linear System
}

\author{
A.L. Cundy ${ }^{1}$, F.M. Hemez ${ }^{2}$, D.J. Inman ${ }^{3}$, G. Park ${ }^{4}$ \\ ${ }^{1}$ Los Alamos National Laboratory, Engineering Sciences and Applications Division, \\ Weapon Response Group, M/S P946 Los Alamos, NM, USA 87544, cundy@lanl.gov \\ ${ }^{2}$ Los Alamos National Laboratory, Engineering Sciences and Applications Division, \\ Weapon Response Group, M/S T006 Los Alamos, NM, USA 87544, hemez@lanl.gov \\ ${ }^{3}$ Center for Intelligent Materials Systems and Structures, Mechanical Engineering \\ Department, Virginia Polytechnic Institute and State University, M/C 0261 Blacksburg, \\ VA, USA 24.061, dinman@vt.edu \\ ${ }^{4}$ Los Alamos National Laboratory, Engineering Sciences and Applications Division, \\ Weapon Response Group, M/S T006 Los Alamos, NM, USA 87544, gpark@lanl.gov
}

\section{Keywords: Damage Identification, Response Surface Metamodels}

\begin{abstract}
Metamodels have been used with success in many areas of engineering for decades but only recently has their use in the field of structural dynamics started to be explored. This research applies the approach of metamodeling to the damage identification problem in structural dynamics. In this case, a metamodel is a reduced order model, constructed by empirically fitting a model to a set of points in the design space. Points in the design space may be chosen using a design of experiments approach. Then a polynomial model is fit to the points using a multiple regression fitting scheme. This empirical fit allows models to be constructed that relate damage parameters of interest (such as stiffness reduction and its location) to measurable output features (e.g. natural frequencies). The method has been demonstrated and its success quantified for a simple linear and nonlinear simulations.
\end{abstract}

\section{Introduction}

The concept of a "metamodel" is introduced as a fast running surrogate model. This is usually a model that will run in minutes on a single processor desktop PC. It could be a traditional reduced order model (as obtained from Guyan Reduction or Craig-Bampton methods), a neural network, or a statistically derived model. The focus of this research will be directed toward statistically derived metamodels, or response surface metamodels for use in damage identification applications, with a simple nonlinear structure as the focus. Figure 1 conceptually demonstrates the area of structural dynamics problems in which it is hoped that response surface methods may be of use. Response surface methods may be employed with low effort and have the potential to be applied to both linear and nonlinear problems.

The concept of a design space is introduced as the set of all possible experiments or simulations that interest the analyst [1]. This is the set that consists of all controllable variables set at all possible levels and associated dependent features of interest. This space may be $n$-dimensional in size. Because the total design space is often prohibitively large, methods have been developed in the literature to efficiently explore it. 


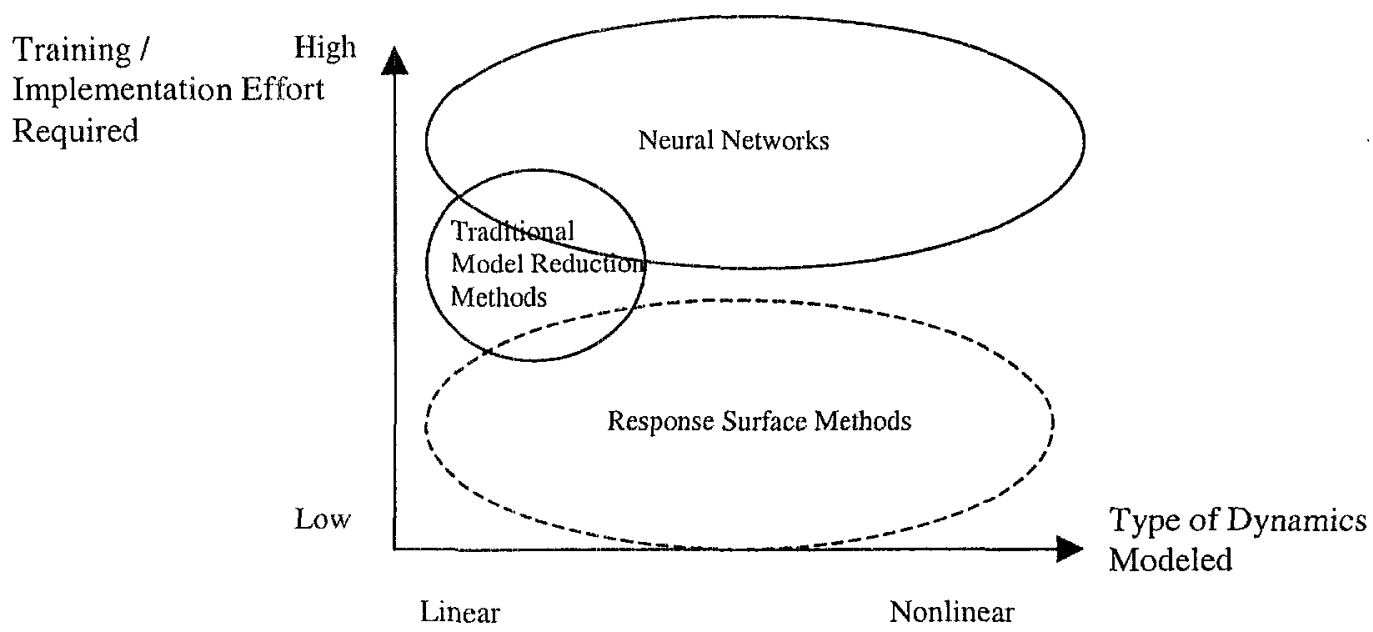

Figure 1 Conceptual plot of the types metamodels and problems for which they are suited.

In the case of response surface metamodels, design of experiments methods (often called response surface methods) are employed. Already popular in the chemical and industrial engineering communities, design of experiments is a statistical method used to "intelligently" determine which simulation or physical experiments should be run when resources are scarce [1]. Design of experiments relies on analysis of variance, or ANOVA, to choose a few points out of the full factorial set that efficiently provide information about the full response space. Metamodels may then be fit to these intelligently chosen data points using standard multiple regression methods resulting in a polynomial model that relates input parameters to output features. This research utilizes a software package called Design Expert by Stat Ease [2]. While these models are empirical in nature, they rely on the expertise of the experimenter for assignment of model input parameters and choice of appropriate output feature(s).

In this work, design of experiments methods are employed to efficiently reduce the size of the design space for a simple linear structure, that is damaged in both a linear and a nonlinear fashion. Multiple regression methods and ANOVA are used to relate damage parameters of interest to measurable output features in a forward sense. Inverse methods are then applied to these relationships to assess the models' capability for predicting damage parameters based on measured output features. Response surface metamodels are capable of detecting damage in nonlinear systems with some quantifiable degree of accuracy.

\section{System Definition}

The system of interest is a simple 5 degree-of-freedom (5DOF hereafter) spring-mass system that has been simulated in MatLab ${ }^{\mathrm{TM}}$ [3] and shown in Figure 2 below. Mass and damping parameters have been held constant at $7 \mathrm{~kg}$ and $1 \mathrm{~kg} / \mathrm{s}$ respectively. In this work, reduced stiffness coefficients constitute "damage" to the system. First damage to 
the linear system will be briefly reviewed. After the ability of response surface metamodels to detect damage in the linear system is established, damage is introduced in the form of nonlinear springs. Response surface metamodels are constructed for this system and the nonlinear damage is detected.
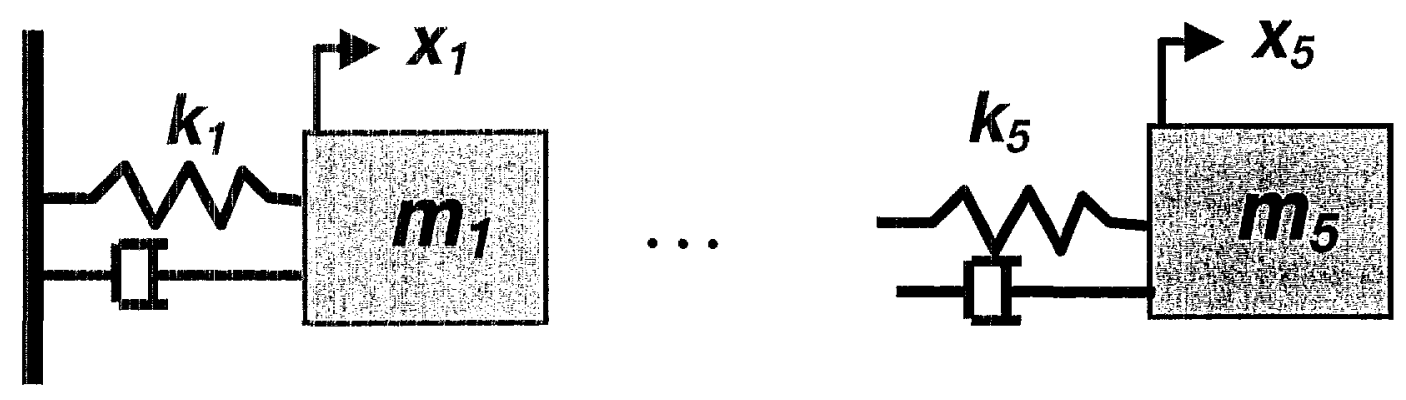

Figure 2 Conceptual drawing of the spring-mass system simulated in MatLab ${ }^{\mathrm{TM}}$.

\section{Damage Identification of the Linear System}

For performance of damage identification using response surface metamodels, choice of damage parameters and measurable output features are of primary importance. In the case of the linear system, relationships between stiffnesses (the chosen damage parameter) and natural frequencies of the system are physical in nature and hence the first five natural frequencies were chosen as measurable output features. The healthy system was defined as all springs at $6000 \mathrm{~N} / \mathrm{m}$. Damage was defined as one spring set at a reduced stiffness value (springs could have a stiffness coefficient of $2000 \mathrm{~N} / \mathrm{m}, 3000$ $\mathrm{N} / \mathrm{m}, 4000 \mathrm{~N} / \mathrm{m}, 5000 \mathrm{~N} / \mathrm{m}$, or $6000 \mathrm{~N} / \mathrm{m}$ ). The entire design space of interest would then consist of 25 simulations, or 5 spring locations and 5 possible values for the spring coefficient. Note that the healthy system was included in the design.

Response surface metamodels were fit to a reduced design space consisting of 9 simulation runs. Ideally this set of 9 runs (shown in Table 1) would adequately represent the full factorial set of 25 runs. For each of these 9 runs, the first five natural frequencies of the system were retained. Then response surface metamodels were fit using multiple regression techniques. An example of one of these surface is shown, in contour format, in Figure 3. These models related controllable input, or damage, parameters (location and stiffness coefficient) to the measurable outputs (natural frequencies). One model was fit per natural frequency due to the functional form of polynomial metamodels. A summary of the polynomial models fit is shown in Table 2 . It can be seen that the $\mathrm{R}^{2}$ values for all of the models indicates very good fit.

Once the relationship between the input parameters and each of the five output features have been established, damage identification is completed by optimizing using the resulting response surfaces in order to do damage identification. That is, knowing the natural frequency values, can the magnitude and location of the reduced stiffness be found? Since natural frequencies were used and they are not highly correlated (they provide linearly independent information), optimization works well. 


\begin{tabular}{|l|l|l|l|l|l|l|l|}
\hline & \multicolumn{2}{|c|}{ Input Parameters } & \multicolumn{5}{|c|}{ Output Features } \\
\hline $\begin{array}{l}\text { Design } \\
\text { Point }\end{array}$ & $\begin{array}{l}\text { Stiffness } \\
{[\mathbf{N} / \mathbf{m}]}\end{array}$ & Location & $\begin{array}{l}\boldsymbol{\omega}_{\mathbf{1}} \\
{[\mathbf{H z}]}\end{array}$ & $\begin{array}{l}\boldsymbol{\omega}_{\mathbf{2}} \\
{[\mathbf{H z}]}\end{array}$ & $\begin{array}{l}\boldsymbol{\omega}_{\mathbf{3}} \\
{[\mathbf{H z}]}\end{array}$ & $\begin{array}{l}\boldsymbol{\omega}_{\mathbf{4}} \\
{[\mathbf{H z}]}\end{array}$ & $\begin{array}{l}\boldsymbol{\omega}_{\mathbf{5}} \\
{[\mathbf{H z}]}\end{array}$ \\
\hline 1 & 2000 & 1 & 6.25 & 20.75 & 35.50 & 47.75 & 55.75 \\
\hline 2 & 6000 & 1 & 8.25 & 24.25 & 38.25 & 49.75 & 56.25 \\
\hline 3 & 2000 & 5 & 8.00 & 19.25 & 31.75 & 45.75 & 55.25 \\
\hline 4 & 6000 & 5 & 8.25 & 24.25 & 38.25 & 49.25 & 56.25 \\
\hline 5 & 2000 & 3 & 7.00 & 22.5 & 32.5 & 48.25 & 51.50 \\
\hline 6 & 6000 & 3 & 8.25 & 24.25 & 38.25 & 49.25 & 56.25 \\
\hline 7 & 4000 & 1 & 7.75 & 22.75 & 37.00 & 48.50 & 56.00 \\
\hline 8 & 4000 & 5 & 8.25 & 23.00 & 35.25 & 47.00 & 55.50 \\
\hline 9 & 4000 & 3 & 8.00 & 23.75 & 36.00 & 49.00 & 53.25 \\
\hline
\end{tabular}

Table 1 Fractional factorial design used for damage identification of the 5DOF system.
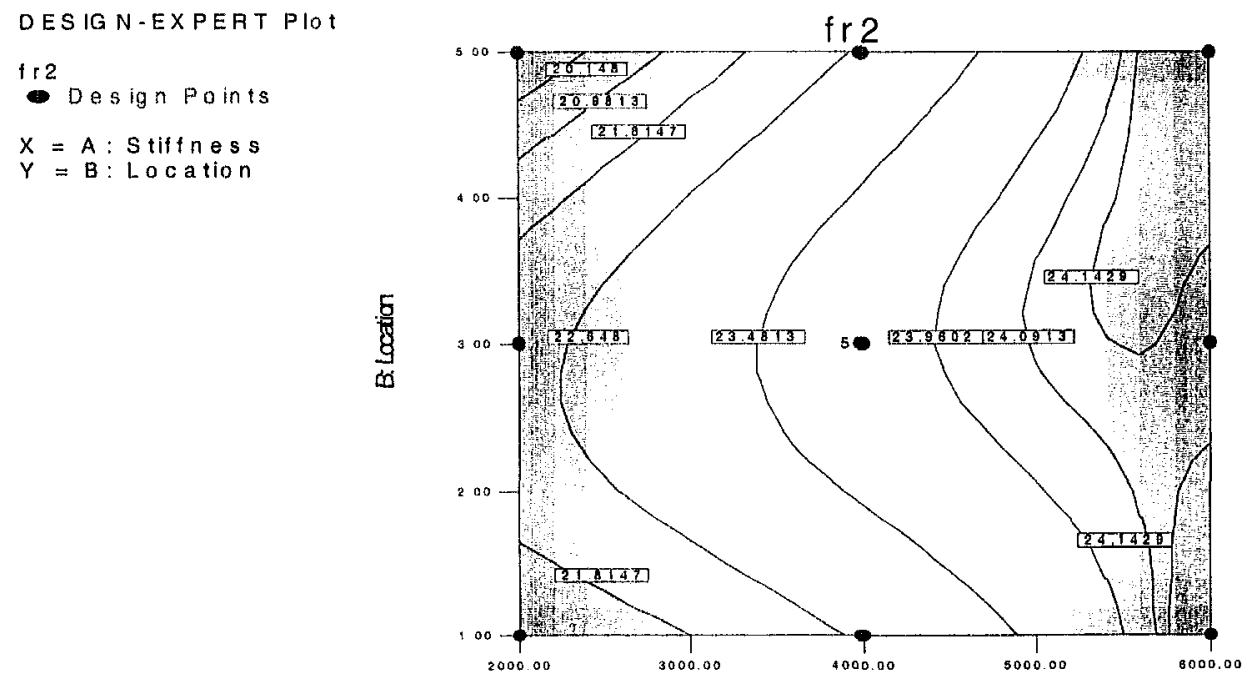

A : $S$ tiffnes $s$

Figure 3 One of the five response surfaces generated for the damage identification problem. Model input parameters are stiffness and location, output in this plot is the second natural frequency.

The optimization procedure used for this set of models was a Nelder-Meade simplex routine embedded in the Stat Ease Design Expert ${ }^{\mathrm{TM}}$ software [2]. It also incorporates the desirability function of Myers and Montgomery [1] which is as follows:

$$
\begin{aligned}
& d=\left(\frac{y-A}{B-A}\right)^{s}, A \leq y \leq B \\
& d=\left(\frac{y-C}{B-C}\right)^{\prime}, B \leq y \leq C
\end{aligned}
$$


The powers $s$ and $t$ weight how quickly the function travels to a maximum desirability of 1 at target value $B$, from the range endpoints of either $A$ or $C$. Desirability is a criterion placed on the measured output values provided. The optimizer then changes the value of the input parameters sought until maximum desirability is achieved near the target output feature values provided. Results are then ranked in order of decreasing desirability. In all cases, the standard followed was to choose the set of input parameters that resulted in the highest desirability rating on the output features.

\begin{tabular}{|c|c|c|}
\hline Frequency & Equation ( $S=$ Stiffness, L=Location) & $\mathrm{R}^{2}$ \\
\hline$\left(\omega_{1}\right)^{-1.6}=$ & $\begin{array}{l}0.036-5.137 \mathrm{E}-003 * \mathrm{~S}-1.798 \mathrm{E}-003 * \mathrm{~L}+3.412 \mathrm{E}-003 * \mathrm{~S}^{2} \\
+7.229 \mathrm{E}-005 * \mathrm{~L}^{2}+4.347 \mathrm{E}-003 * \mathrm{~S} * \mathrm{~L}-2.549 \mathrm{E}-003 * \mathrm{~S} 2 * \mathrm{~L}- \\
7.152 \mathrm{E}-005 * \mathrm{~S}^{*} \mathrm{~L}^{2}\end{array}$ & 1.00 \\
\hline$\omega_{2}=$ & $\begin{array}{l}23.80+0.87 * \mathrm{~S}-0.56 * \mathrm{~S} 2-1.06 * \mathrm{~L}^{2}+0.38 * \mathrm{~S} * \mathrm{~L}- \\
0.38 * \mathrm{~S} 2 * \mathrm{~L}+1.25 * \mathrm{~S} * \mathrm{~L}^{2}\end{array}$ & 1.00 \\
\hline$\omega_{3}=$ & $\begin{array}{l}35.94+2.87 * \mathrm{~S}-0.92 * \mathrm{~L}-0.41 * \mathrm{~S}^{2}+0.34 * \mathrm{~L}^{2}+ \\
0.94 * \mathrm{~S} * \mathrm{~L}-0.56 * \mathrm{~S} * \mathrm{~L}^{2}\end{array}$ & 1.00 \\
\hline$\omega_{4}=$ & $\begin{array}{l}48.93+0.50 * S-0.58 * L-1.01 * L^{2}+0.50 * S * L+ \\
0.75 * S * L\end{array}$ & 0.98 \\
\hline$\omega_{5}=$ & $\begin{array}{l}53.32+2.37 * \mathrm{~S}-0.17 * \mathrm{~L}+0.38 * \mathrm{~S}^{2}+2.26 * \mathrm{~L}^{2}- \\
2.00 * \mathrm{~S} * \mathrm{~L} 2\end{array}$ & 0.99 \\
\hline
\end{tabular}

Table 2 Summary of polynomials generated; units of stiffness and location are scaled between -1 and +1 (coefficients give an idea of term importance).

To test how well the optimization procedure worked, the full factorial set of experiments was optimized (this includes the 9 design points plus 16 points not included in the design, for a total of 25 points). That is, knowing the five natural frequency values (derived from the Matlab ${ }^{\mathrm{TM}}$ simulation), could the change in stiffness (if any) and the location of the change be identified?

Results are shown below in Figure 3 in the form of two plots of actual stiffness or location versus predicted stiffness or location. Ideally these results would plot on a line at $45^{\circ}$ on the plot. Note that all of the mis-identified "Location 1" values corresponded to the "healthy system" (all stiffnesses at $6000 \mathrm{~N} / \mathrm{m}$; these runs were included to see if the healthy system could be identified). It can be observed from Figure 4 that stiffness predictions drift below the ideal line as the stiffness increases. In fact all healthy stiffnesses came back with a value of 5206, Location 1 . If the analyst can be expected to recognize a stiffness prediction of $5206 \mathrm{~N} / \mathrm{m}$ at Location 1 as a "healthy" system, then the damage identification of this system is successful.

If the healthy system is not considered, due to its high location error, most of the errors on stiffness prediction are under $20 \%$. Higher error was expected at the stiffnesses (3000, $5000)$ and locations $(2,4)$ that were not included in the training data set and this was observed. 

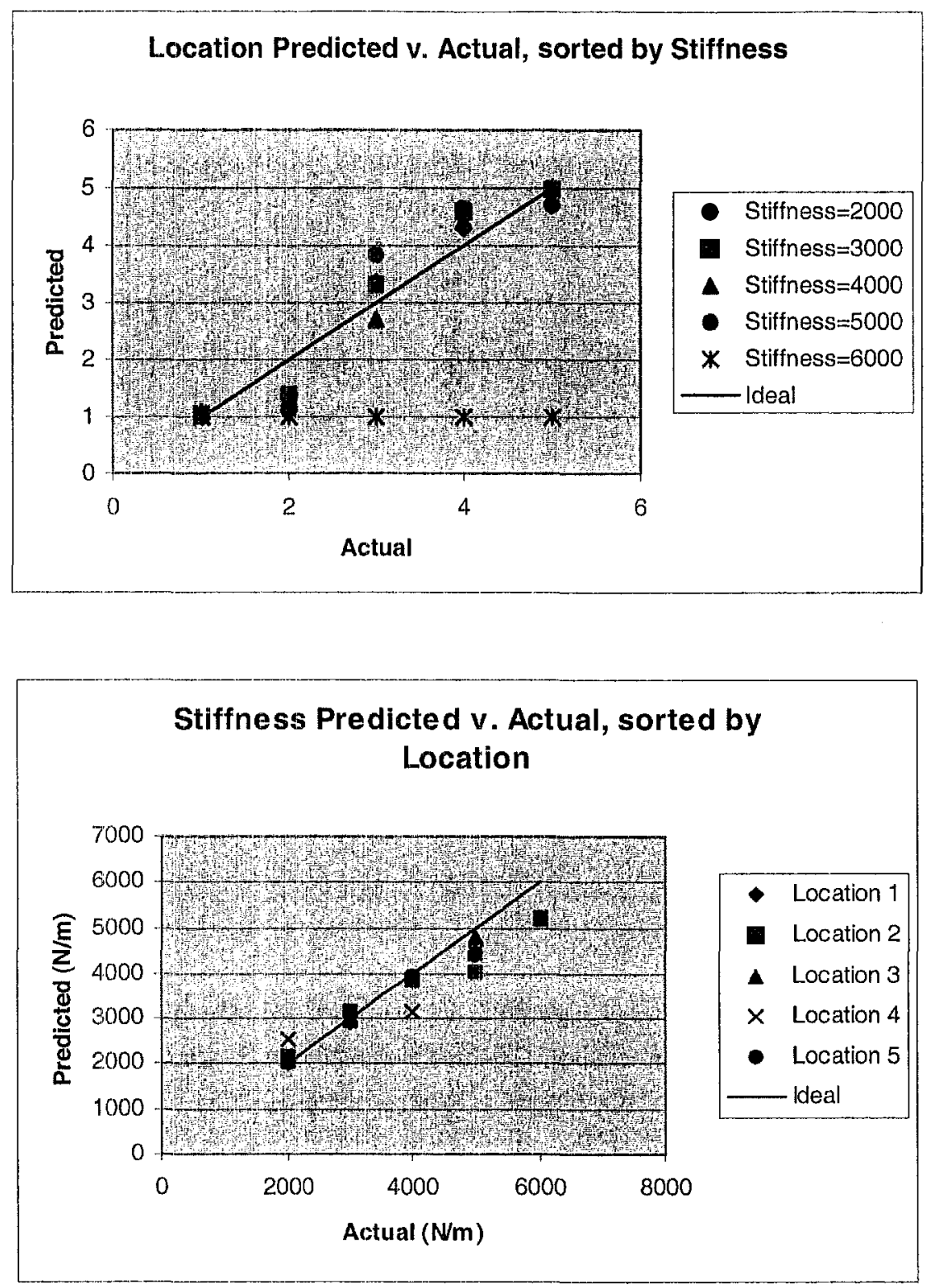

Figure 4 Results of solving the damage identification problem using optimization.

\section{Damage Identification of the Nonlinear System}

Because response surface metamodels were shown to be useful in linear problems it was a natural extension to test their ability to detect nonlinear damage. For this problem, a nonlinear spring was introduced at one of the 5 locations available in the 5DOF springmass system. The nonlinear spring with varying stiffness coefficient was chosen such that it resulted in static displacements that were in the same regime as the linear case.

In the first case, "healthy" was again defined as all linear springs with stiffness coefficient set at $6000 \mathrm{~N} / \mathrm{m}$. Damage was defined as a nonlinear spring replacing a linear 
spring at any of the five locations. Degree of damage was defined as the value of the nonlinear spring coefficient, which could have the following values: $6 \mathrm{e} 6,7 \mathrm{e} 6,8 \mathrm{e} 6,9 \mathrm{e} 6$, $1 \mathrm{e} 7 \mathrm{~N} / \mathrm{m}^{1 / 3}$.

Again, a 9 run fraction of the full factorial design space (25 runs) was used to formulate relationships between controllable inputs and measurable outputs. Because of the presence of nonlinearities in the system, natural frequencies were not chosen, because they were more difficult to identify. Instead maximum displacement and spectral and temporal moments were chosen (See [4] for detailed discussion of the use of moments as extracted features). In this case, the first two spectral moments chosen. The first spectral moment (SE) is how much total energy there is in the broadband sense, and the second spectral moment (ST) is which frequencies contain the most energy (narrow band). Temporal moments have similar meanings, with the first temporal (TE) representing how much total energy there is in a displacement time history, and the second temporal moment (TT) being point in time at which half of the energy has "arrived." Maximum displacement (MD) was also used as an output feature.

Resulting polynomial metamodels are shown below in Table 3 . The input parameters, nonlinear stiffness coefficient and location, have been coded between -1 and +1 , so that variable coefficients give an idea of term importance. Power law transformations of the output features have been used to improve fit in some cases. Note that $\mathrm{R}^{2}$ for the $\mathrm{MD}$ and $\mathrm{SE}$ features are somewhat low $(<0.8)$, potentially making their use in the optimization procedure questionable.

\begin{tabular}{|r|l|l|}
\hline Output Feature & Equation (S=Nonlinear Stiffness, L=Location & $\mathrm{R}^{2}$ \\
\hline $\mathrm{MD}=$ & $0.031-2.033 \mathrm{e}-3^{*} \mathrm{~S}+2.638 \mathrm{e}-3^{*} \mathrm{~S}^{2}-3.817 \mathrm{e}-3^{*} \mathrm{~L}^{2}$ & 0.68 \\
\hline$(\mathrm{SE})^{-1.52}=$ & $69963.58+37130.37 * \mathrm{~S}+27113.25^{*} \mathrm{~L}+10831.69 * \mathrm{~S}^{2}-$ & 0.79 \\
& $12275.83^{*} \mathrm{~L}^{2}$ & \\
\hline $\mathrm{ST}=$ & $6.94-0.075^{*} \mathrm{~S}+4.00 * \mathrm{~L}+0.95^{*} \mathrm{~S}^{2}+0.91^{*} \mathrm{~L}^{2}$ & 0.98 \\
\hline$(\mathrm{TE})^{-1.29}=$ & $784.49+469.6 * \mathrm{~S}+306.14^{*} \mathrm{~L}+163.36^{*} \mathrm{~S}^{2}$ & 0.82 \\
\hline $\mathrm{TT}=$ & $36.07-8.81^{*} \mathrm{~S}-16.45^{*} \mathrm{~L}+6.88^{*} \mathrm{~S}^{*} \mathrm{~L}^{2}$ & 0.97 \\
\hline
\end{tabular}

Table 3 Resulting models for nonlinear damage identification problem.

Results from the damage identification (optimization) procedure are shown below in Figure 5. Optimizations for the design set and the full factorial set have been plotted separately for both location and stiffness coefficient. Location is predicted well (with one exception) for both the design set and the additional points of the full factorial set. Nonlinear stiffness is predicted reasonably well for the design set (errors of less than $15 \%$ ). However stiffness predictions for those points outside of the training set are significantly worse (errors over $20 \%$ in a few cases). 

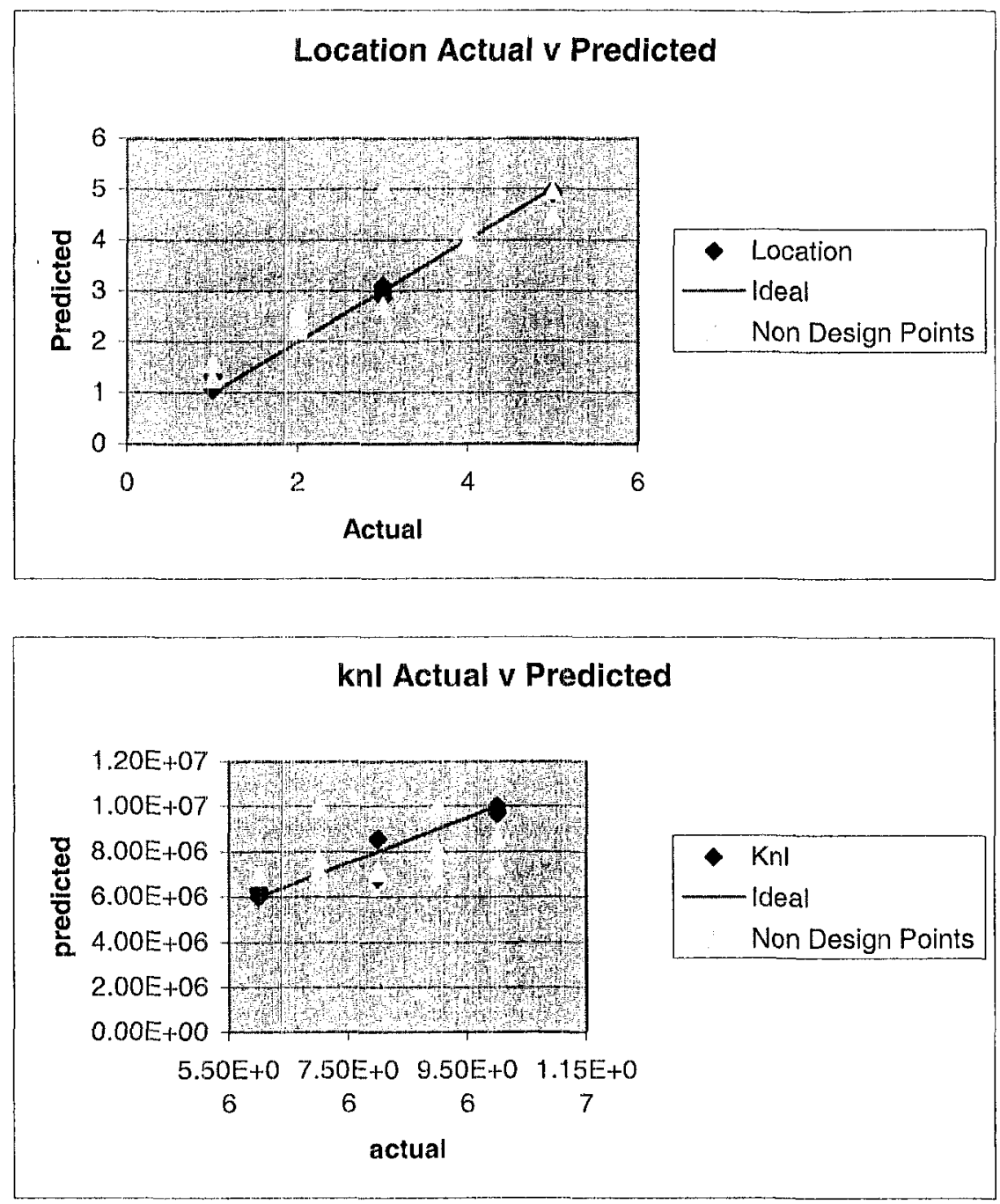

Figure 5 Results from damage identification using response surface metamodels developed for a nonlinear system

\section{Discussion of Results}

For the linear case of the 5DOF system, where damage is represented as reduced stiffness at one of the five locations, response surface metamodels are able to satisfactorily predict stiffness and location values given the simulated natural frequency. This much was accomplished through training on 9 simulations out of 25 possible.

For the case of the nonlinear 5DOF system, in which damage is a nonlinear spring with varying stiffness coefficient inserted at one of the five possible locations, response surface metamodels again show promise in their ability to predict degree and location of damage in this simple, simulated system. In this example, location was predicted satisfactorily for all 25 simulated sets (with one outlier) and stiffness coefficient was predicted well for the design set. Again, this much was accomplished with training on only nine of the 25 possible sets. The use of displacement, temporal and spectral 
moments as output features was investigated and perhaps warrants further investigation. Use of additional training sets and/or features may be necessary to improve the stiffness predictions. 'These improvements are currently being investigated on both this simple 5DOF application, as well as more realistic experimental set-ups that have the potential to be nonlinear in their damage states.

In real-world applications, the analyst will not be able to test the response surface metamodels developed on every possible damage scenario, due to computational and/or experimental costs. In these cases it is recommended that a few scenarios outside of the design set be run in order to assess error that might be incurred "far" from the design points $[5,6]$.

\section{Conclusions}

Response surface metamodels show promise for application in damage identification of both linear and nonlinear dynamic structures. Forward relationships may be generated with relatively few "training" data sets and the inverse formulation may then be applied to conduct damage identification with some quantifiable degree of accuracy. All of these operations may be done on a single processor, desktop PC in seconds making response surface metamodels an attractive option for computationally cheap exploration of the design space of interest.

\section{References}

[1] Myers, R. H., and Montgomery, D. C., 1995, Response Surface Methodology, John Wiley and Sons, Inc., New York, NY.

[2] Stat-Ease, 2000, Design Expert 6.0.5 User's Manual and Software, Minneapolis, $\mathrm{MN}$.

[3] The Mathworks, 2001, Matlab 6.1, Software and Toolboxes, Natick, MA.

[4] Hemez, F. M. and Doebling, S. W., 2003, "From Shock Response Spectrum to Temporal Moments and Vice-Versa," accepted to $21^{\text {st }}$ International Modal Analysis Conference, Kissimmee, FL.

[5] Shinn, R., Hemez, F. M., and Doebling, S. W., 2003, "Estimating the Error in Simulation Prediction Over the Design Space," $44^{\text {th }}$ AIAA/ASME/ASCE/AHS Structures, Structural Dynamics, and Materials Conference, Norfolk, VA.

[6] Cundy, Amanda L. Use of Response Surface Metamodels in Damage Identification of Dynamic Structures. Masters Thesis, Virginia Polytechnic Institute and State University, 2002. 